\title{
Method for Aureole Estimation Refinement Through Comparisons Between Observed Aureole and Estimated Aureole Based on Monte Carlo Ray Tracing
}

\author{
Kohei Arai ${ }^{1}$ \\ Graduate School of Science and Engineering \\ Saga University \\ Saga City, Japan
}

\begin{abstract}
Method for aureole estimation refinement through comparisons between observed aureole and estimated aureole based on Monte Carlo Ray Tracing: MCRT is proposed. Through some experiments, it is found that the proposed method does work for refinement of aureole estimation. The experimental results also show the proposed method is validated through comparison with empirical aureole estimation equation which is proposed by Shebrooke University research group.
\end{abstract}

Keywords-Monte Carlo method; Ray tracing method; Aureole; aerosol; optical depth; solar diffuse; solar direct

\section{INTRODUCTION}

In order to improve estimation accuracy of aerosol refractive index, it is necessary to improve estimation accuracy and measuring accuracy of aureole, and solar diffuse irradiance. It is not so difficult to improve solar direct irradiance because it is large enough for measurements. Meanwhile, it is not so easy to improve measuring and estimating aureole and solar diffuse irradiance because these are quit small.

For many years, research scientists proposed their method for measurement and estimation of aureole which results in empirical equation of aureole. One of those is the well known empirical equation proposed by Shebrooke University research group.

On the other hand, it would be better to improve or refine the estimated aureole by using empirical equation. Aureole can be estimated with some atmospheric codes, or Monte Carlo Ray Tracing: MCRT method. Measured aureole can be refined by using estimated aureole derived from MCRT in the sense of least square means. The proposed method is based on this idea. Through comparisons the refined aureole and empirically estimated aureole, the proposed method can be validated.

The following section describes research background and the proposed method followed by some experimental data. Then conclusion is described together with some discussions.

\section{PROPOSED MODEL}

\section{A. Theoretical Background}

The solar aureole is a region of enhanced brightness within about $20^{\circ}$ of the sun's disk, due to the predominant forwardscattering effect of atmospheric aerosols. The solar aureole theory and its use for determining aerosol size distributions also has been discussed in detail by Deirmendjian(1959). Here, we calculated aureole irradiance using MS(multiply scattering) approximation proposed by M.A.Box et.al(1981). They assumed that the solar aureole irradiance was essentially due to SS(single scattering) by aerosols and molecules, and MS by molecules alone. Moreover, they also taken into account the effect of the surface reflectance in the computation of the aureole irradiance. P.Romanov et al.(1999) summarized an empirical formula of aureole irradiance based on the method proposed by M.A.Box et.al. It is shown as follows,

$I_{\text {aur }}(\Theta, \lambda)=\frac{F_{0}(\lambda)}{\mu_{0}} \exp \left(-\frac{\tau(\lambda)}{\mu_{0}}\right)\left(\tau_{m o l}(\lambda) P_{m o l}(\Theta, \lambda)+\tau_{s c a}(\lambda) P_{a e r}(\Theta, \lambda)+\Delta_{m s}(\Theta, \lambda, A)\right)$

where $),(\lambda \Theta a$ aurI is the aureole irradiance measured at scattering angle $\Theta,),(\lambda \Theta$ molP and $),(\lambda \Theta$ aerP are the Rayleigh and aerosol phase functions, respectively. $)(\lambda \tau s c a$ is the aerosol scattering optical depth. $),(A m s \lambda \Theta \Delta$ is a term that represents the contribution of the effects of multiple scattering and reflection from the surface in solar aureole. It can be written as follows,

$$
\Delta_{m s}(\Theta, \lambda, A)=\tau_{A}(\lambda, A) P_{m o l}\left(0^{0}, \lambda\right)+P_{m o l}(\Theta, \lambda) t_{m s}
$$

where

$$
\tau_{A}(\lambda, A)=A \tau_{2} /\left(1-A \tau_{3}\right)
$$$$
\tau_{2}=1.34 \tau_{s s} \mu_{0} /\left(1.0+0.22\left(\tau_{s s} / \mu_{0}\right)^{2}\right),
$$$$
\tau_{3}=0.9 \tau_{s s}-0.92 \tau_{s s}^{2}+0.54 \tau_{s s}^{3} \text {, }
$$$$
t_{m s}=0.02 \tau_{s s}+1.2 \tau_{s s}^{2} / \mu_{0}^{0.25} \text {, }
$$$$
\tau_{s s}=\tau_{R}(\lambda)+\tau_{s a}(\lambda)
$$ 
A is the surface reflectance and $s s \tau$ is the total scattering optical depth.

Figure 1 shows a comparison of the calculated result between aureole irradiance from MS model and diffuse irradiance from the method of successive orders of scattering. The aureole irradiance was calculated by the aureole model described as above in an angle range of the forward scattering in which the azimuth angles are from $0^{\circ}$ to $30^{\circ}$ and the zenith angle is the same as the solar zenith angle. Meanwhile, the diffuse irradiance was calculated in all azimuth angles from $0^{\circ}$ to $180^{\circ}$ by the method of the successive orders of scattering. In the forward scattering, we found that it was quite different of calculated results between the diffuse irradiance and the aureole irradiance. This is because that it exist not only scattering but also diffraction when light passes particle in the forward direction. It results in generating a region of enhanced brightness in forward direction. From now on, we removed the unrealistic part of diffuse irradiance, and remained the corresponding exact estimates of the aureole irradiance in the forward scattering.

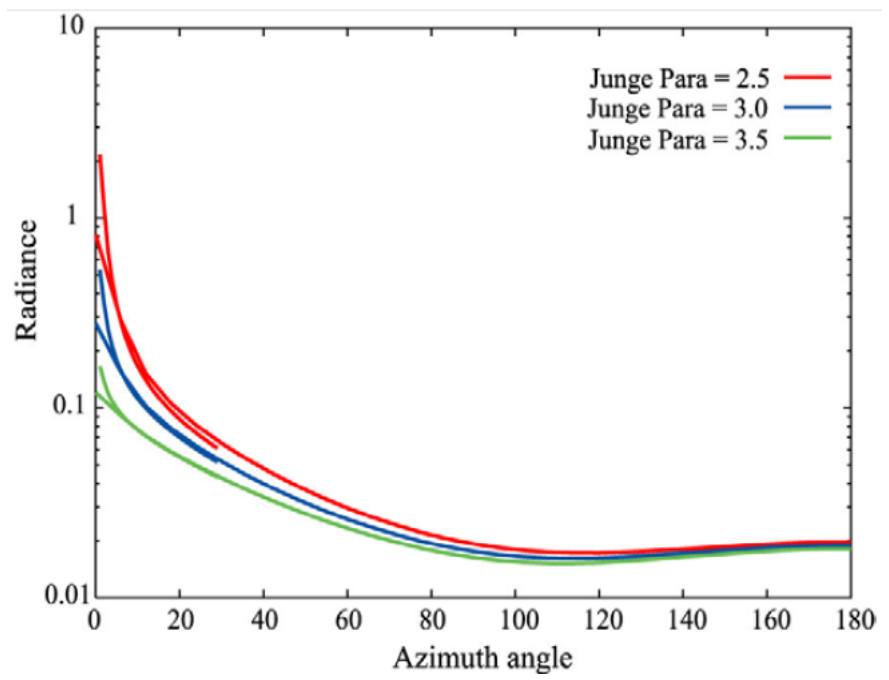

Fig. 1. Comparison the Aureole Irradiance from MS Model and Diffuse Irradiance from the Method of Successive Orders of Scattering

\section{B. Shebrooke Model}

The estimation method for the refractive index and the size distribution of aerosol proposed by P.Romanovs of the Sherbrooke University based on linear inversion method is summarized as follows. The aerosol refractive index and size distribution are retrieved by minimizing the squared difference between the measured data and the simulated data of direct solar irradiance at surface (or aerosol optical depth), downward diffuse irradiance at surface and solar peripheral irradiance (aureole) based on the plane-parallel atmospheric model. This model also considered molecular Rayleigh scattering and Mie scattering which is dependence on the aerosol refractive index and size distribution.

First,

$F=H \varphi+e$ is defined where $\mathrm{F}$ is the vector of the deviations of the measured data, that is,

$$
F=\left(\begin{array}{l}
I_{d i r}-I_{d i r}^{*} \\
I_{d i f}-I_{d i f}^{*} \\
I_{a u r}-I_{a u r}^{*}
\end{array}\right)
$$

[*] denotes the measurement data. $\mathrm{H}$ is a Jacobian, and can be expressed as,

$$
H=\left(\begin{array}{ccccc}
I_{\text {dir }}^{\prime}(v 1) & \ldots & I_{\text {dir }}^{\prime}(v m) & I_{\text {dir }}^{\prime}(\kappa) & I_{d i r}^{\prime}(\eta) \\
I_{d i f}^{\prime}(v 1) & \ldots & I_{d i f}^{\prime}(v m) & I_{d i f}^{\prime}(\kappa) & I_{d i f}^{\prime}(\eta) \\
I_{\text {aur }}^{\prime}(v 1) & \ldots & I_{\text {aur }}^{\prime}(v m) & I_{\text {aur }}^{\prime}(\kappa) & I_{\text {aur }}^{\prime}(\eta)
\end{array}\right)
$$

where Idir is Solar direct irradiance, IdifI is Solar diffuse irradiance and Iaur is aureole irradiance. $I_{\text {dir }}^{\prime}, I_{\text {dif }}^{\prime}, I_{\text {aur }}^{\prime}$ individually indicate a factor of the matrix when the relation between the direct and diffuse solar irradiance and aureole, and the refractive index and size distribution of aerosol is represented as a matrix.

It is also expressed that $E=E_{0} \exp \left(-\tau / \mu_{0}\right)$, where E0 is the exoatmospheric solar irradiance. According to previous equation, we can replace the direct solar irradiance with aerosol optical depth. From now on, discussion will be focused on aerosol optical depth instead of the direct solar irradiance. $0 E$

In the formulation of the inverse problem the vector $\phi$ is defined as follows,

$$
\begin{aligned}
& \varphi^{T}=\left\{\varphi_{v 1} \ldots \varphi_{v m}, \varphi_{\kappa}, \varphi_{\eta}\right\} \\
& \varphi_{v m}=s-s^{*} \\
& s \in v 1, \ldots, v m, \kappa, \eta
\end{aligned}
$$

where $m v v, \ldots, 1$ represent size distribution of aerosol per air column. $\eta \kappa$, are the real part and the imaginary part of the refractive index.

Because the aerosol optical depth, diffuse solar irradiance and aureole can be measured on the ground, the Eq.(5.2) can be given. It is possible to estimate the unknown parameters of the refractive index and the size distribution of aerosol in the Eq. (5.3). However, because the number of the unknown parameters that represent the size distribution and the real part and the imaginary part of the refractive index is far more than the number of the measured data, it is an ill-posed problem. Hence, it can be solved with commonly used inversion solution based on minimizing norm of estimation error then,

$$
\varphi=H^{T}\left[H H^{T}\right]^{-1} F
$$


It is well known that the retrieval accuracy of the minimized norm method is not good enough because the number of the unknown parameters is more than it of the equations given. Since the influence of the imaginary part of the refractive index on aureole is so small that the accuracy improvement of the imaginary part of refractive index cannot be expected from this method.

Assuming the aerosol size distribution as Junge distribution, according to Eq.(3.22), the size distribution can be represented as only one Junge parameter $(a)$. Therefore, the Eq.(5.3) can be rewritten as,

$$
H=\left(\begin{array}{ccc}
\tau_{a e r}^{\prime}(a) & \tau_{a e r}^{\prime}(\kappa) & \tau_{\text {aer }}^{\prime}(\eta) \\
I_{d i f}^{\prime}(a) & I_{d i f}^{\prime}(\kappa) & I_{d i f}^{\prime}(\eta) \\
I_{a u r}^{\prime}(a) & I_{a u r}^{\prime}(\kappa) & I_{a u r}^{\prime}(\eta)
\end{array}\right)
$$

The number of the unknown parameters is three which is totally identical to the number of the equations to be given, so that

$$
\left(\begin{array}{l}
\kappa-\kappa^{*} \\
\eta-\eta^{*} \\
a-a^{*}
\end{array}\right)=\left(\begin{array}{lll}
\tau_{a e r}^{\prime}(a) & \tau_{a e r}^{\prime}(\kappa) & \tau_{a e r}^{\prime}(\eta) \\
I_{d i f}^{\prime}(a) & I_{d i f}^{\prime}(\kappa) & I_{d i f}^{\prime}(\eta) \\
I_{a u r}^{\prime}(a) & I_{a u r}^{\prime}(\kappa) & I_{a u r}^{\prime}(\eta)
\end{array}\right)^{-1}\left(\begin{array}{c}
\tau_{a e r}-\tau_{a e r}^{*} \\
I_{d i f}-I_{d i f}^{*} \\
I_{a u r}-I_{a u r}^{*}
\end{array}\right)
$$

The refractive index and Junge parameter can be retrieved simultaneously

In accordance with Shebrooke University group, aureole can be expressed in equation (1), empirically.

$$
\begin{aligned}
L= & \Phi_{0} \sec \theta_{0} \exp \left(-\tau_{T} \sec \theta_{0}\right) \\
& {\left[\left(\tau_{M}+\tau_{M S}\right) P_{M}+\tau_{P S} P_{P}+\tau_{A} P_{M}\left(0^{\circ}\right)\right] }
\end{aligned}
$$

where

$$
\begin{aligned}
& \tau_{A}=\frac{A \tau_{2}\left(\tau_{S S}, \mu_{0}\right)}{1-A \tau_{3}\left(\tau_{S S}\right)} \\
& \tau_{M S}=0.02 \tau_{S S}+1.2 \tau_{S S}^{2} \mu_{0}^{-1 / 4} \\
& \tau_{A}=\frac{A \tau_{2}\left(\tau_{S S}, \mu_{0}\right)}{1-A \tau_{3}\left(\tau_{S S}\right)} \\
& \tau_{2}=1.34 \tau_{S S} \mu_{0}\left[1.0+0.22\left(\tau_{S S} / \mu_{0}\right)^{2}\right] \\
& \tau_{3}=0.9 \tau_{S S}-0.92 \tau_{S S}^{2}+0.54 \tau_{S S}^{3} \\
& \tau_{S S}=\tau_{M}+\tau_{P S} \quad \mu_{0}=\cos \theta_{0}
\end{aligned}
$$

Thus aureole can be estimated with the above empirical equation.

\section{Methiod for Refinement of Measured Aureole}

Figure 2 shows the proposed method for refinement of measured aureole with estimated aureole based on MODTRAN. Method for aureole estimation refinement through comparisons between observed aureole and estimated aureole based on Monte Carlo Ray Tracing: MCRT is proposed.

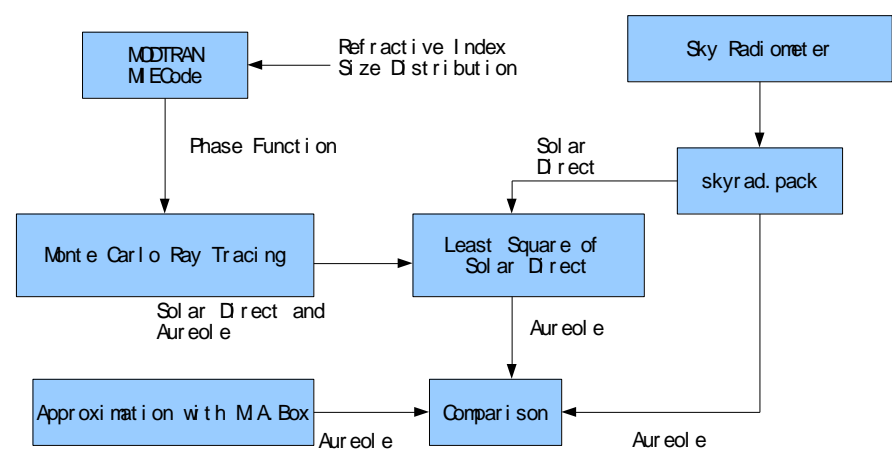

Fig. 2. shows the proposed method for refinement of measured aureole with estimated aureole based on MODTRAN.

Essentially, aureole can be measured with sky radiometer which is shown in Figure 3.

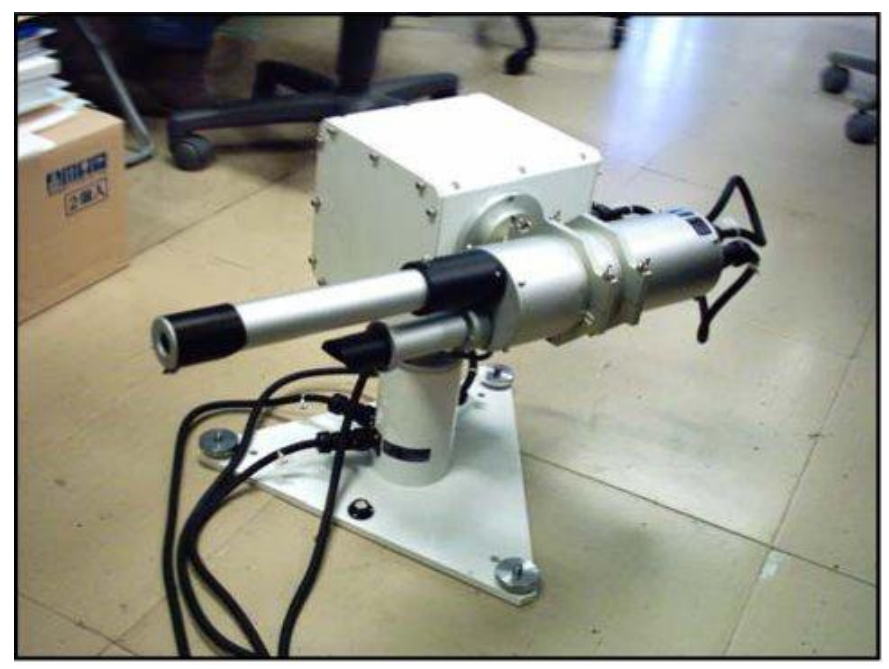

Fig. 3. Outlook of the sky radiometer manufactured by PREDE Co. Ltd. Japan

POM-1 of Sky radiometer manufactured by Prede Co. Ltd. Japan is used for measurements of solar direct and diffuse irradiance including aureole. In order for improvement of irradiance measurement accuracy, the proposed method minimizes the difference between measured and model derived aureole. Mie code of MODTRAN of atmospheric code allows estimating phase function (scattering angle dependency) which depends on aerosol refractive index and size distribution. MCRT, on the other hand, allows estimation of refractive index and size distribution with the other atmospheric parameters including optical depth. Meanwhile, solar direct, diffuse including aureole can be measured with POM-01. Through minimizing square difference between the measured and estimated solar direct irradiance, estimation 
accuracy for aureole can be improved. After that aureole can be refined through comparison between empirical aureole calculated by using model proposed by Box et al. and measured aureole.

\section{Monte Carlo Ray Tracing Model}

Figure 4 shows flow chart of MCRT.

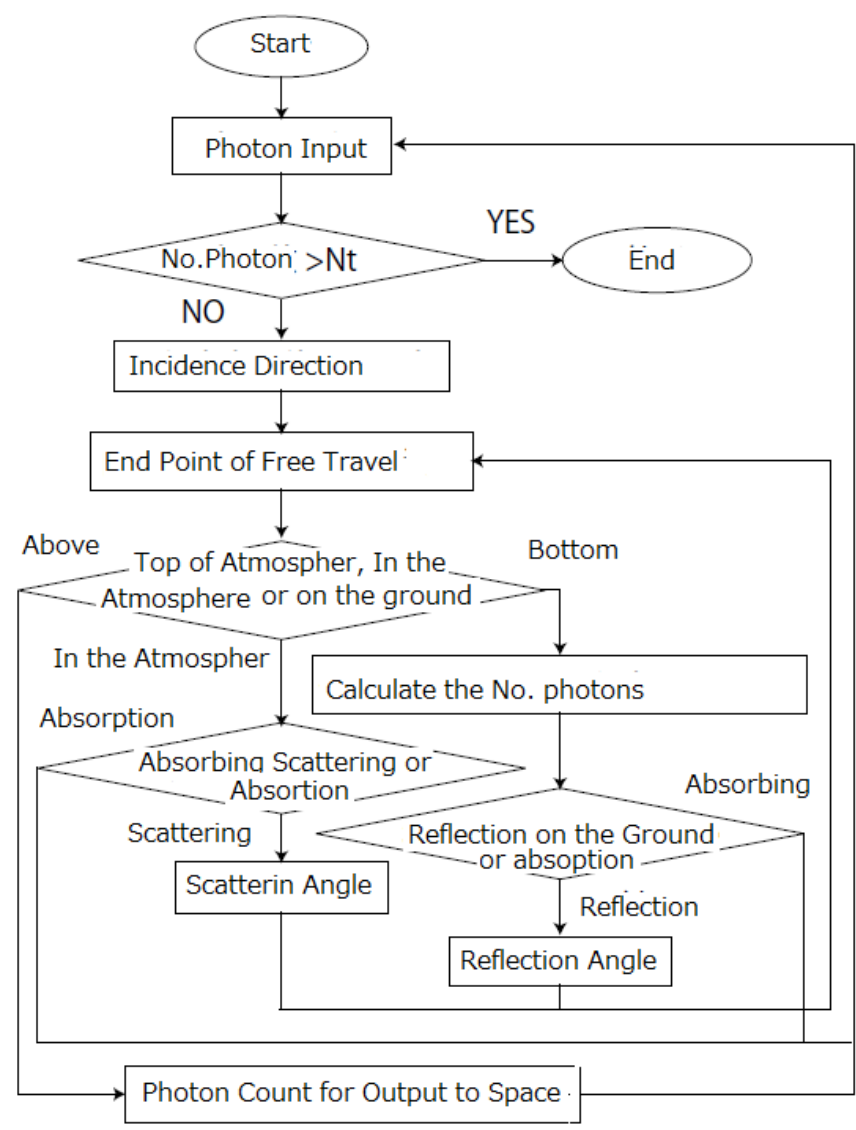

Fig. 4. Flowchart of MCRT

Scattering angle is defined in Figure 5.

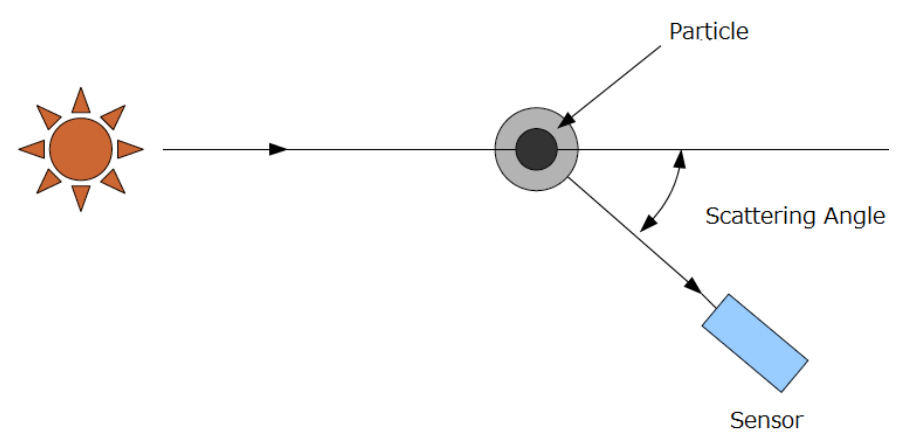

Fig. 5. Definition of scattering angle

Examples of phase function is shown in Figure 6.

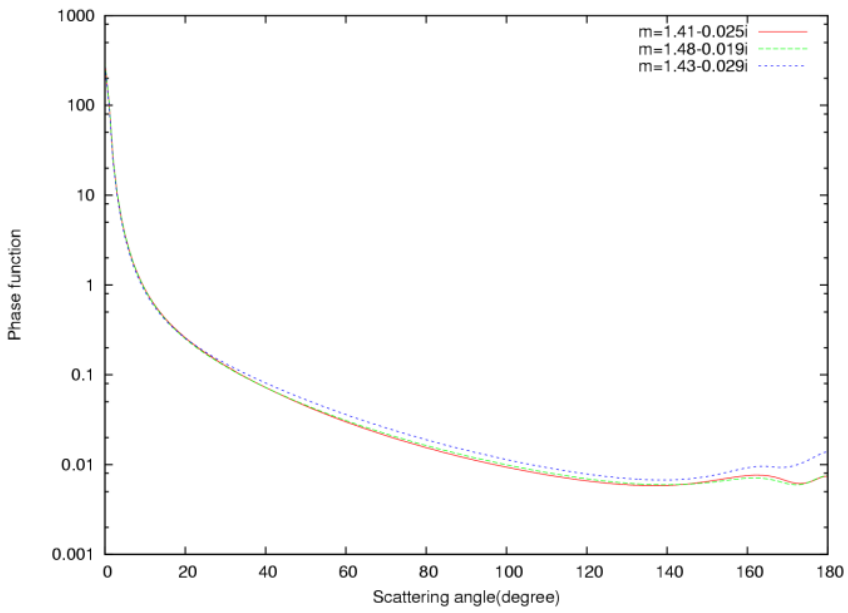

Fig. 6. Examples of phase functions

Refractive indexes of the examples are as follows,

$\mathrm{m}=1.41-0.025 \mathrm{i}$

$\mathrm{m}=1.48-0.019$

$\mathrm{m}=1.43-0.029 \mathrm{i}$

A photon is put in the simulation cell from the top of the cell with the incidence angle that depends on the specified solar zenith angle. The position of which the photon is put in is changed by time by time in accordance with the uniformly distributed random numbers.

Depending on the optical depth of the atmosphere, free travel length $L$ of photon is determined as follows,

$$
\begin{aligned}
& L=-L_{0} \log (\text { Rnd }) \\
& L_{0}=\frac{h}{\tau_{\text {all }}}
\end{aligned}
$$

where $L_{0}$ is called free travel length, denoting the average distance of interaction of a photon from one position to another. Rnd is uniformly distributed random numbers ranges from 0 to $1 . h$ denotes the physical height of the atmosphere $\left(50 \mathrm{~km}\right.$ in this case) while $\tau_{\text {all }}$ denotes the optical depth of the atmosphere which is determined as follows,

$$
\tau_{\text {all }}=\tau_{\text {aero }}+\tau_{\text {mol }}
$$

where the subscript aero is associated with aerosols while mol with molecules. Here, it is assumed that atmosphere consists of aerosols and air molecules. Because the wavelength in concern ranges from 450 to $1050 \mathrm{~nm}$ so that optical depth of ozone and water vapors are assumed to be negligible except $936 \mathrm{~nm}$ of water vapor absorption band. A small absorption due to ozone is situated from 500 to $650 \mathrm{~nm}$ around. 
The photon meets aerosol particles or molecule when the photon travels in the atmosphere then scattering due to the aerosols or molecules occurs. The probability of the collision to the aerosols or molecules depends on their optical depths. If the endpoint of photon travel is in the atmosphere, the photon meets aerosol or molecule. The probability of the photon meets aerosol is $\tau_{\text {aero }} / \tau_{\text {all }}$ while that of the photon meets molecule is $\tau_{\text {mol }} / \tau_{\text {all }}$. In accordance with the phase function of aerosols or molecules, the photon is scattered. Strength of scattering as a function of scattering angle $\theta$ is determined by the phase function, $P(\theta)$, the Rayleigh for molecules, equation (4) and Heyney-Greestein function, equation (5) (it is just an approximation function of which the phase function is monotonically decreasing) for aerosols. Actual phase function can be determined with MODTRAN 4.0 of Mie code with the measured refractive index of aerosols through field experiments. By using uniformly distributed random numbers, scattering direction is determined. The phase function as $P(\theta)$, where $\theta$ is the angle between the incident direction and the scattering direction.

For molecules, the Rayleigh phase function is as follows,

$$
P(\theta)=(3 / 4)\left(1+\cos ^{2} \theta\right)
$$

while that for aerosols, we use the Heyney-Greenstein approximation function of the following,

$$
P(\theta)=\frac{1-g_{\lambda}^{2}}{\left(1+g_{\lambda}^{2}-2 g_{\lambda} \cos \theta\right)^{3 / 2}}
$$

where $g_{\lambda}$ is the asymmetry factor of the aerosol phase function which depends on the wavelength of the radiation and the compositions, sizes, and the shapes of the aerosol particles.

In the calculation of TOA radiance, the number of photons, $N$ which comes out from the top of the atmosphere within the angle range which corresponds to the Instantaneous Field of View: IFOV of the sensor in concern is used thus the normalized TOA radiance, Rad is determined as follows,

$$
\operatorname{Rad}=\frac{\mu_{0} \mu \Delta \mu}{2}\left(\frac{N}{N_{\text {total }}}\right)
$$

where $\mu_{0}=\cos \theta_{0}, \mu=\cos \theta, \theta_{0}$ is the solar zenith angle and $\theta$ is a viewing solid angle. $\Delta \mu$ is a view solid angle, i.e., FOV (field of view).

$N_{\text {total }}$ is the number of photons which are put in the cell in total. If you multiply solar irradiance to Rad in unit of $\left(\mathrm{W} / \mathrm{m}^{2} / \mathrm{str} / \mu \mathrm{m}\right)$, then the TOA radiance in the same unit is calculated.

\section{EXPERIMENTS}

\section{A. Measured Data}

Table 1 shows measured data which are obtained at Saga University, Japan at 11:35 Local Time in Japan on April 25
2004. It was fine day. Solar zenith angle was 22.5 degree at that time.

TABLE I. PARAMETRRS MEASURED AT EXPERIMENTS

\begin{tabular}{|l|l|l|l|l|l|}
\hline Wavelength(um) & 0.400 & 0.500 & 0.675 & 0.870 & 1.020 \\
\hline Real_Refractive_Index & 1.430 & 1.410 & 1.430 & 1.490 & 1.450 \\
\hline Imaginary_Refractive_Index & 0.028 & 0.017 & 0.024 & 0.006 & 0.018 \\
\hline Aerosol_Optical_Depth & 0.295 & 0.220 & 0.170 & 0.140 & 0.125 \\
\hline Molecule_Optical_Depth & 0.360 & 0.143 & 0.024 & 0.015 & 0.008 \\
\hline
\end{tabular}

Solar irradiances estimated with Sky Rad Pack, empirical equation (Box), and Monte Carlo is shown in Figure 7. Although the proposed method utilizing Monte Carlo Ray Tracing simulation shows better accuracy rather than Sky Rad Pack and empirical equation for shorter wavelength, the proposed method shows poorer accuracy than the conventional methods for longer wavelength. The reason for this is atmospheric optical depth is too thin.

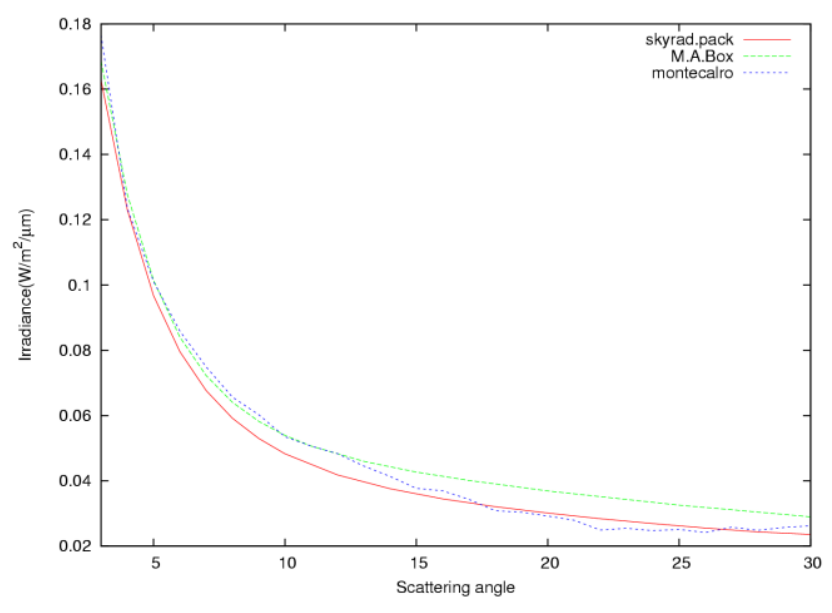

(a) $400 \mathrm{~nm}$

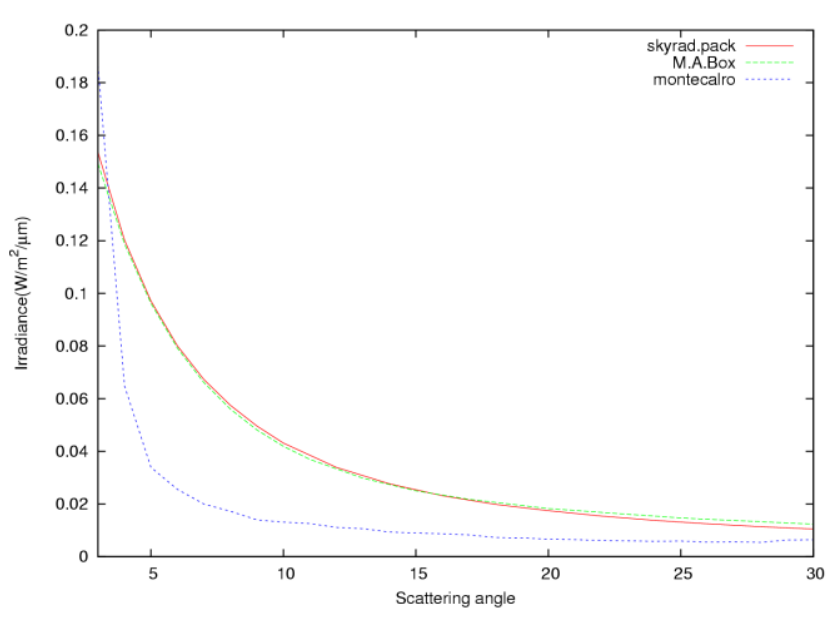

(b) $500 \mathrm{~nm}$ 

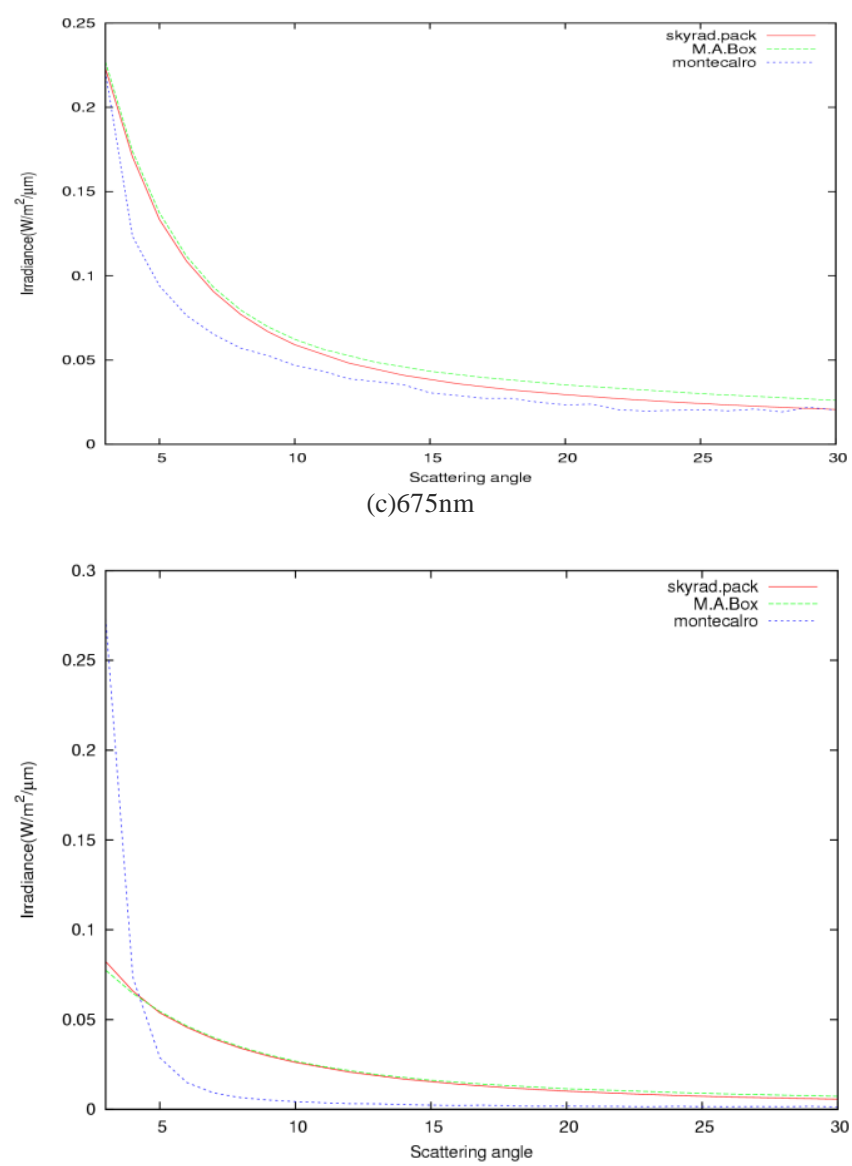

(d) $875 \mathrm{~nm}$

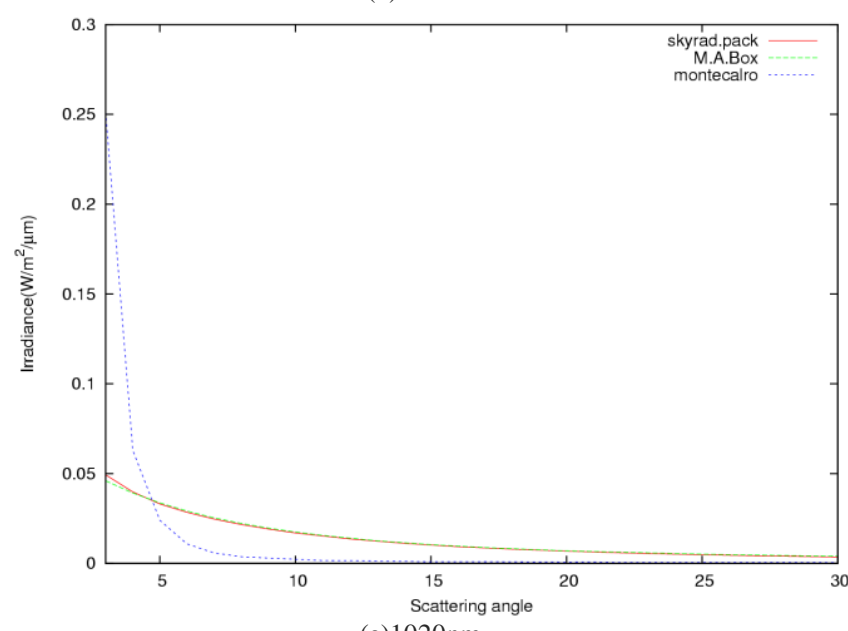

(e) $1020 \mathrm{~nm}$

Fig. 7. Solar irradiances estimated with (a) Sky Rad Pack, (b) empirical equation (Box) and (c) Monte Carlo simulation.

Estimation error is defined with equation (15).

$\operatorname{Error}(\%)=\sqrt{\frac{\sum\left(\frac{X_{n}-x_{n}}{X_{n}}\right)^{2}}{N}} \times 100$
Estimation errors of real and imaginary parts of refractive index, aerosol optical depth, molecule optical depth and surface reflectance are shown in Table 2.

TABLE II. ESTIMATION ERRORS OF REAL AND IMAGINARY PARTS OF REFRACTIVE INDEX, AEROSOL OPTICAL DEPTH, MOLECULE OPTICAL DEPTH AND SURFACE REFLECTANCE

\begin{tabular}{|l|l|l|l|l|l|}
\hline Wavelength(um) & 0.400 & 0.500 & 0.675 & 0.870 & 1.020 \\
\hline Refractive_Index & $0.9 \%$ & $22.7 \%$ & $67.0 \%$ & $78.5 \%$ & $81.0 \%$ \\
\hline Aerosol_Optical_Depth & $26.4 \%$ & $57.9 \%$ & $83.3 \%$ & $93.6 \%$ & $96.1 \%$ \\
\hline Molecule_Optical_Depth & $17.4 \%$ & $69.8 \%$ & $99.0 \%$ & $97.1 \%$ & $95.6 \%$ \\
\hline Surface_Reflectance & $27.4 \%$ & $50.3 \%$ & $82.3 \%$ & $90.4 \%$ & $92.5 \%$ \\
\hline
\end{tabular}

\section{B. Sensitivity Analysis}

Sensitivity analysis is conducted for validation of the proposed method. Designated parameters for sensitivity analysis are shown in Table 3. Meanwhile, Table 4 shows the results from the sensitivity analysis.

TABLE III. PARAMETERS FOR SENSITIVITY ANALYSIS

\begin{tabular}{|c|c|c|c|c|}
\hline \multicolumn{2}{|c|}{} & Mn. & Max. & St ep \\
\hline \multirow{2}{*}{ Ref r act i ve I ndex } & Real & 1.350 & 1.600 & 0.010 \\
\cline { 2 - 5 } & I magi nary & 0.000 & 0.030 & 0.001 \\
\hline \multirow{2}{*}{ Opt i cal Dept h } & Aer osol & 0.000 & 1.000 & 0.050 \\
\cline { 2 - 5 } & Nol ecul e & 0.000 & 1.000 & 0.050 \\
\hline \multicolumn{2}{|c|}{ Surf ace Pef I ect ance } & 0.000 & 1.000 & 0.050 \\
\hline
\end{tabular}

TABLE IV. THE RESULTS FROM THE SENSITIVITY ANALYSIS.

\begin{tabular}{|l|c|c|c|c|c|c|}
\hline \multicolumn{2}{|c|}{ Whivel engt $\mathrm{h}(\mu \mathrm{m})$} & 0.400 & 0.500 & 0.675 & 0.870 & 1.020 \\
\hline \multicolumn{2}{|c|}{ Ref r act i ve I ndex } & $4.9 \%$ & $-12.0 \%$ & $-64.5 \%$ & $-72.3 \%$ & $-78.0 \%$ \\
\hline \multirow{2}{*}{ qtical Depth h } & Aer osol & $-11.8 \%$ & $-47.2 \%$ & $-80.8 \%$ & $-87.5 \%$ & $-93.1 \%$ \\
\cline { 2 - 7 } & Nol ecul e & $-2.8 \%$ & $-59.1 \%$ & $-96.5 \%$ & $-90.9 \%$ & $-92.6 \%$ \\
\hline \multicolumn{2}{|l}{ Sur f ace Ref I ect ance } & $-12.8 \%$ & $-39.6 \%$ & $-79.8 \%$ & $-84.3 \%$ & $-89.5 \%$ \\
\hline
\end{tabular}

It is found that only real and imagery parts of refractive index at $400 \mathrm{~nm}$ shows relatively good estimation accuracy. The other parameters show under estimated situation. One of the reasons for this is that the number steps for real and imaginary parts are greater than the others. Estimation accuracy at $1020 \mathrm{~nm}$ is not so good relatively. There are small absorptions due to water vapor and ozone at the $1020 \mathrm{~nm}$ slightly.

Estimation accuracy for the shorter wavelength is better than that for longer wavelength. Therefore, the sensitivity for the shorter wavelength is greater than that for the longer wavelength. 


\section{Relation between estimation errors of aureole and refractive index}

Aureole estimation accuracy may affect to estimation of refractive index. Relation between estimation error of aureole and refractive index is investigated with the parameters listed in Table 2. The relation between aureole and real part of refractive index is shown in Figure 8 (a) while that between aureole and imaginary part of refractive index is shown in Figure 8 (b).
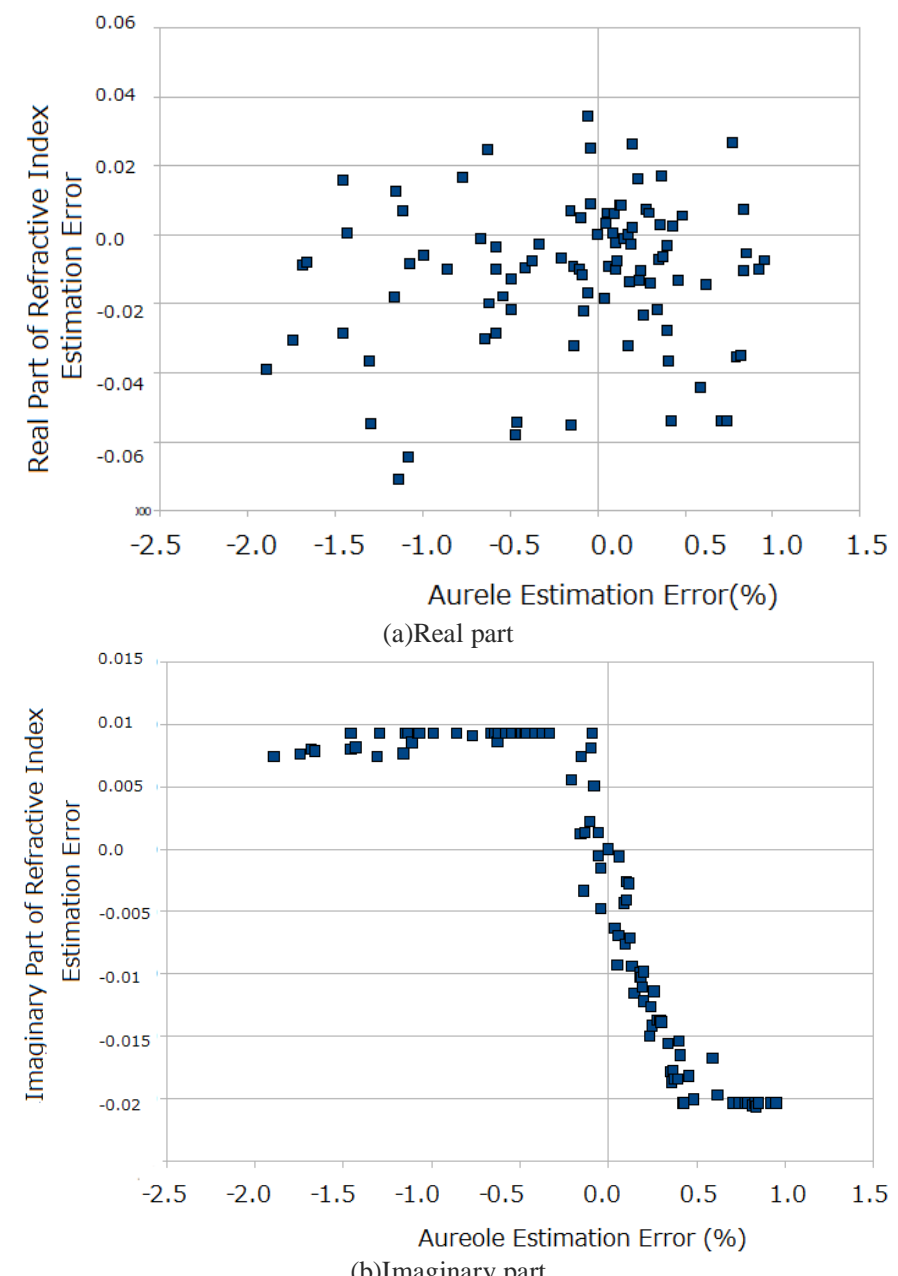

Fig. 8. Relation between aureole estimation error and that of refractive index.

The relation between aureole estimation error and that of real part of refractive index shows somewhat positive trend, correlation coefficient is not high though. Meanwhile, the relation between aureole estimation error and that of imaginary part of refractive index shows somewhat systematic and shows negative trend. Imaginary part of refractive index is corresponding to absorption. Therefore, negative trend of the relation is reasonable.

\section{CONCLUSION}

Method for aureole estimation refinement through comparisons between observed aureole and estimated aureole based on Monte Carlo Ray Tracing: MCRT is proposed. Through some experiments, it is found that the proposed method does work for refinement of aureole estimation. The experimental results also show the proposed method is validated through comparison with empirical aureole estimation equation which is proposed by Shebrooke University research group.

Although the proposed method utilizing Monte Carlo Ray Tracing simulation shows better accuracy rather than SkyRad-Pack and empirical equation for shorter wavelength, the proposed method shows poorer accuracy than the conventional methods for longer wavelength. The reason for this is atmospheric optical depth is too thin.

The relation between aureole estimation error and that of real part of refractive index shows somewhat positive trend, correlation coefficient is not high though. Meanwhile, the relation between aureole estimation error and that of imaginary part of refractive index shows somewhat systematic and shows negative trend. Imaginary part of refractive index is corresponding to absorption. Therefore, negative trend of the relation is reasonable.

\section{ACKNOWLEDGMENT}

The author would like to thank Mr. Yu Odeishi for his efforts through experiments and simulations.

\section{REFERENCES}

[1] Arai K., Xing Ming Lian, aerosol refractive index and size distribution estimation usinbg solar direct and diffuse irradiance based on simulated annealing, Journal of Japan Society of Remote Sensing, 23, 2, 1120,2003 .

[2] K.Arai and Xing Ming Liang, Method for estimation of refractive index and size distribution of aerosol using direct and diffuse solar irradiance and aureole by means of simulated annealing, Advances in Space Research, Vol.32, No.11, pp.2165-2174, 2003.

[3] K.Arai and Xing Ming Liang, Method for estimation of refractive index and size distribution of aerosol using direct, diffuse and aureole by means of simulated annealing. Proceddings of the COSPAR (Committee on Space Research) Congress, p.10 (Solicited Paper), Texas, USA, Oct., 2002.

[4] K.Arai, Y.Iisasa and Xing Ming Liang, Aerosol parameter estimation with changing observation angle of ground based polarization radiometer, Proc. of the COSPAR Congress (Paris), A-00579,A3.1-003704, July 2004.

[5] K.Arai and Xing Ming Liang, Characterization of aerosols in Saga city areas in Japan with direct and diffuse solar irradiance and aureole observations, Proc.of the COSPAR Congress(Paris), A-00582, A1.10130-04, July 2004.

[6] Arai, K., Xing Ming Liang, Aerosol refractive index and size distribution estimation using solar direct and diffuse irradiance by means of nonlinear insion method, Proceedings of the $33^{\text {rd }}$ General Assembly of the Japan Remote Sensing Society, 145-146, 2002.

[7] Aarai K., Xing Ming Lian, Aerosol refractive index and size distribution estimation using solar direct, aureole, and solar diffuse irradiance based on nonlinear inversion, Proceedings of the 34th General Assembly of the Japan Remote Sensing Society, 209-210, 2003.

[8] Romanov, P., Norman T.O'Neill and A.Royer, Simultaneous retrival of aerosol refactive index and particle size distribution from ground-based measurements of direct and scattered solar radiation, Applied Optics, Vol. 38, No. 36, pp.7305-7320,1999.

[9] Aarai K., Fundamental Theory on Remote Sensing, Gakujutsu-ToshoShuppan Publishing Co. Ltd., 2001

[10] Multispectral Remote Sensing of Earth's Atmosphere, N. Takeuchi Edt., Gakkai-Shuppan-Senta Publishing Co. Ltd., 2001.

[11] Guang-Yu Shi, Ground-based measurements of aerosol parameters, Institute of atmospheric Physics, Chinese Academy of Science, G-0063 second progress report, 1998 
[12] I.Lenoble, Radiative transfer in scattering and absorption atmospheres, A. DEEPAK Publishing, 1985.

[13] Shunlin liang, Quantitative remote sensing of land surface, John Wiley \& Sons, Inc, 2004.

[14] Box,M.A. and A.DEEPAk, An approximation to multiple scattering in earth's atmosphere: Almucantar irradiance formulation, Journal of atmospheric science, Vol.38, pp.1037-1048,1981.

[15] eirmendjian,D., Theory of solar aureole, Part I , application to atmospheric models, ann. Geophys., vol.15, pp.218-249,1959.

[16] McClatchey,R.A.,R.W.Fenn,J.E.A.Selby,F.E.Volz and J.S.Garing, Optical properties of the atmosphere( 3 ed.). AFCRL Envirn.Res.Papers No.411, pp108.,1972.

[17] Arai,K and Y.Minematsu, Image restoration based on maximum entropy method with parameter estimation by annealing, Journal of the Japanese photogrametry and remote sensing society, Vol. 37, No. 2, pp.1522,1998 .

\section{AUTHORS PROFILE}

Kohei Arai, He received BS, MS and PhD degrees in 1972, 1974 and 1982, respectively. He was with The Institute for Industrial Science and Technology of the University of Tokyo from April 1974 to December 1978 also was with National Space Development Agency of Japan from January, 1979 to March, 1990. During from 1985 to 1987, he was with Canada Centre for Remote Sensing as a Post Doctoral Fellow of National Science and Engineering Research Council of Canada. He moved to Saga University as a Professor in Department of Information Science on April 1990. He was a councilor for the Aeronautics and Space related to the Technology Committee of the Ministry of Science and Technology during from 1998 to 2000 . He was a councilor of Saga University for 2002 and 2003. He also was an executive councilor for the Remote Sensing Society of Japan for 2003 to 2005 . He is an Adjunct Professor of University of Arizona, USA since 1998. He also is Vice Chairman of the Commission "A" of ICSU/COSPAR since 2008. He wrote 30 books and published 492 journal papers 